\title{
Effective Reduction of Goresky-Kottwitz-MacPherson Graphs
}

\author{
Charles Cochet
}

\section{CONTENTS}

1. Introduction

2. Definition and First Properties of GKM Graphs

3. Classical Examples of GKM Manifolds and Graphs

4. Reduction of a 3-Independent Abstract 1-Skeleton

5. Quantization and Reduction Commute

6. Programs

7. Test of Programs

Acknowledgments

References
2000 AMS Subject Classification: Primary 53D20, 68R10

Keywords: Hamiltonian manifold, symplectic reduction, quantization, cohomology, $K$-theory, GKM graph, Cayley graph, Johnson graph, graph reduction
The Goresky-Kottwitz-MacPherson (GKM) graph is a combinatorial analogue of a compact connected symplectic manifold with a Hamiltonian action of a compact torus. This graph has been intensively studied by Guillemin and Zara, who discovered analogues in graph theory of classical results such as: symplectic reduction and "quantization and reduction commute." In this paper, we describe the implementation of algorithms illustrating their results.

\section{INTRODUCTION}

In 1988, Thomas Delzant [Delzant 88] opened a new path between Hamiltonian geometry and the world of convex polytopes. For any symplectic compact connected manifold with Hamiltonian effective action, the dimension of the manifold is at least twice the dimension of the torus and the image of the manifold by the moment map is a convex polytope. Moreover, if this dimension is exactly twice that of the torus, then the convex polytope (named Delzant's polytope) characterizes up to isomorphism the

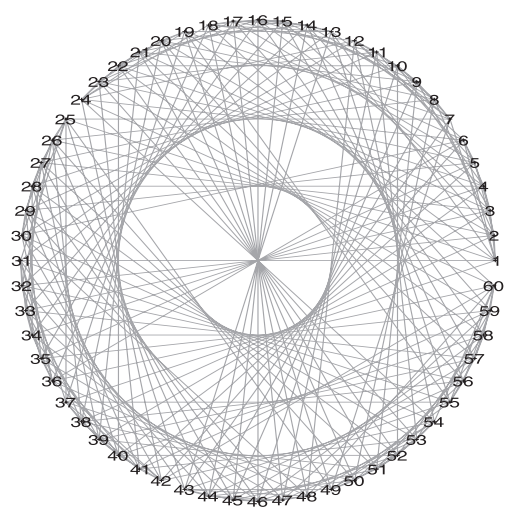

FIGURE 1. GKM graph of the manifold $(G L(3, \mathbb{C}) / B)$ $\times G_{2,5}(\mathbb{C})$ (screenshot from our program). 

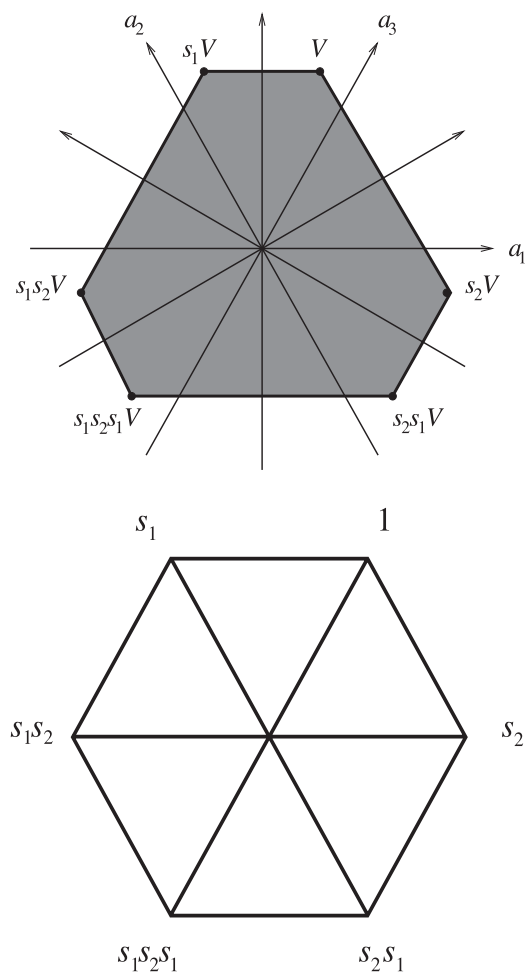

FIGURE 2. Image by the moment map of the coadjoint orbit $U(3) \cdot V$ and GKM graph of the flag manifold $U(3) /\{$ diagonal matrices $\}$.

Hamiltonian manifold. In other words, all data from the Hamiltonian manifold is stored in this polytope.

Demazure [Demazure 70] introduced the notion of toric manifold (see also [Audin 91, Guillemin 94]). A certain subcategory of these manifolds, containing, in particular, projective spaces, satisfy hypotheses of Delzant's theorem. Unfortunately, many interesting manifolds do not fulfill these drastic conditions, for example, Grassmannian and flag manifolds. Thus, during the passage from these manifolds to their associated polytope loss of data occurs. Hence, we want to know how to encode all data from a compact connected manifold with action of a torus of any dimension.

Goresky, Kottwitz, and MacPherson proved [Goresky et al. 98] that the ring of equivariant cohomology of certain compact connected manifolds-GKM manifolds, which we will discuss later - can be computed with tools from graph theory. Among GKM manifolds are toric manifolds and homogeneous spaces $G / H$, where $G$ is a compact connected group and $H$ a subgroup of $G$ of same rank.

Guillemin and Zara highlighted a graph associated to each GKM manifold. This graph, with edges

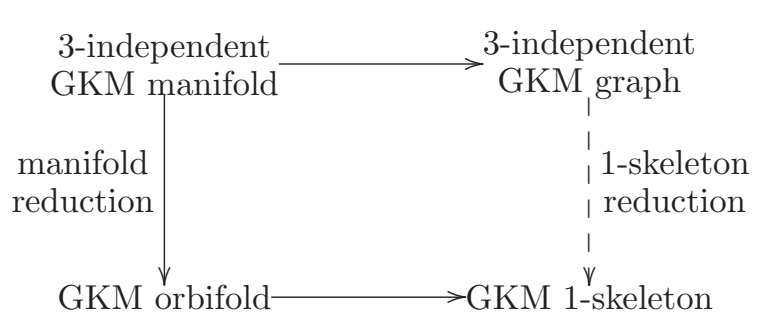

FIGURE 3. Relationship between manifold reduction and 1-skeleton reduction.

oriented and labeled by an axial vector, is called the Goresky-Kottwitz-MacPherson (GKM) graph, or 1-skeleton [Guillemin and Zara 99]. For example, in the Hamiltonian case, this graph takes into account the fact that images of fixed points by the moment map are linked not only by edges in the sense of polytopes (intersection of facets), but also sometimes by edges "inside" the polytope (see Figure 2).

Guillemin and Zara then "forgot" the underlying manifold: the abstract 1-skeleton was born. Since then, they have investigated properties of this new object [Guillemin and Zara 00, Guillemin and Zara 01a, Guillemin and Zara 01b]). In graph theory, they found many analogues of notions of symplectic geometry: orientation, cohomology, $K$-theory, quantization. They also proved in GKM theory counterparts of several classical theorems from symplectic geometry, like Atiyah-Bott-Berline-Vergne's localization theorem [Berline and Vergne 82, Atiyah and Bott 84] and Jeffrey-Kirwan's theorem [Jeffrey and Kirwan 95].

Under certain hypotheses, Guillemin and Zara computed the reduction of an abstract 1-skeleton by a onedimensional torus and at a regular value of a moment map of the graph. The reduction remains an abstract 1-skeleton. This graph operation imitates the reduction of a manifold, so that the reduction of the graph of a manifold is the graph of the reduced manifold (when this makes sense). See Figure 3.

Probably, the most fascinating part of their research in the framework of the reduction of a GKM graph by a one-dimensional torus is as follows. In fact, the invariant character of a $K$-theory element equals a character built only with data coming from the reduced graph (and from the $K$-theory element), called the reduced character. This result is the analogue in graph theory of the assertion "quantization and reduction commute" from the symplectic world [Meinrenken and Sjamaar 99]. In addition, while the invariant character of a $K$-theory element 

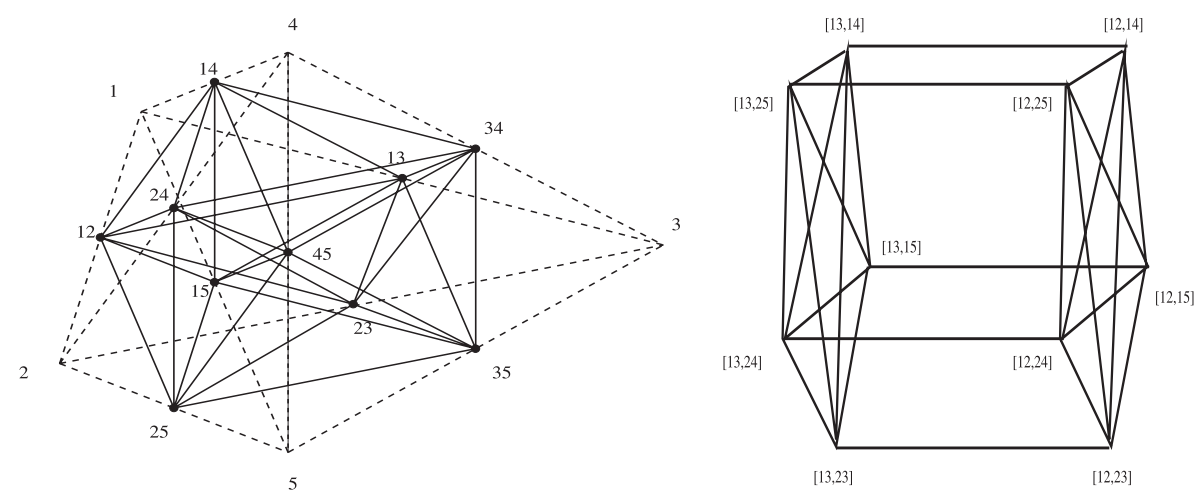

FIGURE 4. GKM graphs of $G_{1,5}(\mathbb{C})=\mathbb{P}^{4}(\mathbb{C})$ (left, dashed), and $G_{2,5}(\mathbb{C})$ (left, plain) and its reduction along $\xi=$ $(0,1,2,3,-6)$ at $c=7 / 2$ (right).

is a rather big polynomial, the reduced character is a condensed rational fraction.

Reduction of a 1-skeleton is a fastidious task. After low-dimensional examples we have to face intractable computations. For example, the graph of the Grassmannian of complex 2-planes in $\mathbb{C}^{5}$ is 6 -valent and has ten vertices (hence 30 edges). Its reductions by the torus generated by $\xi=(0,1,2,3,-6)$ are 5 -valent and possess $6,10,12$, and 14 vertices (hence 15, 25, 30, and 35 edges). See Figure 4. Computer science is a great help, when studying nontrivial examples.

Consequently, I implemented, in the language of the computer algebra system Maple, the reduction of a 1-skeleton, using a program named reduction.mws. The output is not only data of the reduced graph (vertices, edges, and axial vectors), but also a graphical representation of the result.

Similarly, the computation and the storage of the invariant character are intractable even for small examples. For instance, the dilatation of the $K$-theory element implies an impressive growth of the number of terms of the character. For the $K$-theory element $\Theta(p)=e^{2 i \pi \theta_{p}}$ of the manifold $\mathbb{P}^{3}(\mathbb{C})$ and for the one-dimensional torus with infinitesimal generator $\xi=(1,2,-1,-2)$, invariant characters $\chi\left(\Theta^{n}\right)^{H}$ for $n=1,10,100$, and 1,000 have respectively $1,12,867$, and 83,667 monomials. This is why I also implemented the computation of the reduced character of a $K$-theory element of a GKM graph, in a program named character.mws. The output is a sum of rational fractions with constant size whatever the dilatation of the $K$-theory element is.

My two programs (reduction.mws and character .mws) contain a library of examples. Procedures generate Grassmannians $G_{k, n}(\mathbb{C})$ and the cycle with $4 N$ vertices. The flag manifold $U(3) /\{$ diagonal matrices $\}$ (whose re- duction is a GKM hypergraph) is also available. A procedure performs the product of 1-skeleta.

These two programs aim to make the understanding of GKM graphs easier. I implemented them using Maple, a widespread software whose language is quite understandable. There are lots of commentaries along with both programs, so that a curious user may understand internal procedures. The source code is freely available and may be modified. The independence of subroutines allows adapting programs to one's needs.

Programs can be downloaded at http://www.math. jussieu.fr $/ \sim$ cochet $/$.

This paper is organized as follows. Sections 2-5 contain a survey of results of Guillemin and Zara about GKM theory. Only Sections 6-7 are original. Section 2 introduces GKM graphs. Fundamental examples (Grassmannians and flag manifolds) are described in Section 3. Section 4 features the reduction of abstract GKM graphs. Section 5 is devoted to the analogue for GKM graphs of the theorem "quantization and reduction commute." Examples are included, for the sake of clarity. Section 6 contains the description of my programs. Finally, we examine several tests of programs in Section 7, and discuss their implementation and performances.

\section{DEFINITION AND FIRST PROPERTIES OF GKM GRAPHS}

\subsection{GKM Graph of a Manifold}

Let $G=\left(S^{1}\right)^{n}$ be the $n$-dimensional torus with Lie algebra $\mathfrak{g}=\mathbb{R}^{n}$. Let $\left(e_{j}\right)_{j}$ be the canonical basis of $\mathbb{R}^{n}$. Its dual basis is $\left(\theta_{j}\right)_{j} \subset \mathfrak{g}^{*}$. Let $u_{j}=e^{2 i \pi \theta_{j}}(1 \leq j \leq n)$. We denote by $\mathbb{Z}_{G}^{*}=\mathbb{Z}^{n}$ the set of weights of linear forms $\lambda=\sum_{j=1}^{n} \lambda_{j} \theta_{j}$ with $\lambda_{j} \in \mathbb{Z}$. The set $\mathbb{Z}_{G}^{*}$ is the weight lattice for $G$. Let $\xi=\left(\xi_{1}, \ldots, \xi_{n}\right)$ be an element of $\mathfrak{g}$. 
Definition 2.1. A compact connected manifold $M$ of dimension $2 d$ on which $G$ acts is a GKM manifold if:

1. the set $M^{G}$ of fixed points of $M$ under the action of $G$ is finite;

2. $M$ has an almost complex $G$-invariant structure; and

3. for all $p \in M^{G}$, weights $\alpha_{p, i} \in \mathbb{Z}_{G}^{*}(1 \leq i \leq d)$ of the action of $G$ on the tangent space $T_{p} M$ are pairwise linearly independent.

From a GKM manifold $(M, G)$, let us build a $d$-valent graph $\Gamma$. The set of its vertices is by definition $M^{G}$. Let $H_{p, i}$ be the subtorus of $G$ of dimension $n-1$ and of Lie algebra $\mathfrak{h}_{p, i}=\operatorname{ker}\left(\alpha_{p, i}\right) \subset \mathfrak{g}$.

Proposition 2.2. The connected component $E^{p, i}$ of $M^{H_{p, i}}$ containing $p$ is isomorphic to the complex space $\mathbb{P}^{1}(\mathbb{C})$, that is, to the Riemann sphere $S^{2}$. The action of $G$ on $E^{p, i}$ restricts to the action of $S^{1}$ on $S^{2}$ by rotation.

In particular, $E^{p, i}$ contains two fixed points: $p$ and, say, $q$. Then $p$ and $q$ are linked by an edge, denoted by $e=[p, q]$. Denote by $\bar{e}$ the edge $[q, p]$. Let $\alpha_{p, e}=$ $\alpha_{p, q}=\alpha_{p, i}$ (respectively $\alpha_{q, \bar{e}}=\alpha_{q,[q, p]}=\alpha_{q, j}$ ) be the weight of the action of $G$ on $T_{p} E^{p, i}$ (respectively $T_{q} E^{p, i}$ ). Then $\alpha_{p, e}=-\alpha_{q, \bar{e}}$. Thus for each weight $\alpha_{p, i}$ we get one complex space $\mathbb{P}^{1}(\mathbb{C})$ and one $q=q(p, i)$ linked to $p$ by an edge. Repeating this process with every fixed point $p$, we obtain a $d$-valent graph $\Gamma$.

Denote by $E(p)$ the set of edges pointing out from $p$, and by $N(p)$ the set of neighbors of $p$. Let $V_{\Gamma}$ be the set of vertices of the graph $\Gamma$. The incidence relation $I_{\Gamma}$ of $\Gamma$ is the set of couples $(p, e)$, where $p \in V_{\Gamma}$ and $e \in E(p)$.

The application $\alpha: I_{\Gamma} \longrightarrow \mathbb{Z}_{G}^{*} \backslash\{0\}$ defined by $\alpha(p, e):=\alpha_{p, e}$ is called the axial function.

Definition 2.3. The couple $(\Gamma, \alpha)$ is the GKM graph or GKM 1-skeleton of $M$.

The axial function satisfies the properties in Proposition 2.4.

\section{Proposition 2.4.}

1. If $e=[p, q]$ is an edge of $\Gamma$, then $\alpha_{p, q}=-\alpha_{q, p}$.

2. Let $\left(p_{i}\right)=N(p)$. Then weights $\alpha_{p, p_{i}}$ are pairwise linearly independent.
3. Let $e=[p, q] \in E(p)$ and $\mathfrak{g}_{e}=\operatorname{ker}\left(\alpha_{p, q}\right)$. Let $\left(p_{i}\right)=$ $N(p) \backslash\{q\}$ (respectively $\left.\left(q_{i}\right)=N(q) \backslash\{p\}\right)$. Then one can renumber the $q_{i}$ 's such that $\alpha_{p, p_{i}}=\alpha_{q, q_{i}}+$ $c_{e, i} \alpha_{p, q}$ for integers $c_{e, i}$.

Remark 2.5. In general $G$ does not act faithfully on $M$. Let $\mathfrak{z}$ be the Lie algebra of the kernel $Z$ of the representation of $G$ in $\operatorname{Aut}(M)$. Then the axial function takes its values in $\mathfrak{z}^{\perp}$. We say that $M$ is toric when its dimension is twice the dimension of the torus $G / Z$.

The category of GKM Hamiltonian compact connected manifolds is a source of examples of manifolds with easily computed GKM graphs.

Remark 2.6. The construction of a GKM graph does not require a symplectic structure. Thus, whereas a manifold can have several symplectic structures, it has only one GKM graph.

\subsection{Abstract GKM Graph}

In this section, we formalize the notion of a GKM 1-skeleton of a GKM manifold $M$ and study its properties, independently of the existence of $M$.

Fix a $d$-valent graph $\Gamma$.

Definition 2.7. An axial function on $\Gamma$ is an application $\alpha: I_{\Gamma} \longrightarrow \mathfrak{g}^{*} \backslash\{0\}$ such that:

1. If $e=[p, q]$ is an edge of $\Gamma$, there exist $m_{e}>0$ and $m_{\bar{e}}>0$ such that $m_{e} \alpha_{p, e}=-m_{\bar{e}} \alpha_{q, \bar{e}}$.

2. Let $\left(p_{i}\right)=N(p)$. Then vectors $\alpha_{p, p_{i}}$ are pairwise linearly independent.

3. Let $e=[p, q]$ be an edge of $\Gamma$ and $\mathfrak{g}_{e}=\operatorname{ker}\left(\alpha_{p, e}\right)$. Let $\left(p_{i}\right)=N(p) \backslash\{q\}$ and $\left(q_{i}\right)=N(q) \backslash\{p\}$. Then one can renumber $q_{i}$ 's so that there exist $\tau_{e, i}>0$ and $c_{e, i} \in \mathbb{R}$ such that $\alpha_{p, p_{i}}=\tau_{e, i} \alpha_{q, q_{i}}+c_{e, i} \alpha_{p, q}$.

The couple $(\Gamma, \alpha)$ is called an abstract 1-skeleton.

When $m_{e}=m_{\bar{e}}, \tau_{e, i}=1$, and $\alpha$ takes its values in the weight lattice $\mathbb{Z}_{G}^{*}$, we speak of an abstract $G K M$ 1-skeleton or an abstract GKM graph. This is consistent with Section 2.

Proposition 2.8. [Guillemin and Zara 01a] If $(\Gamma, \alpha)$ is an abstract GKM graph, there exist a complex manifold $M$ and an action of $G$ on $M$ such that $(\Gamma, \alpha)$ is the $G K M$ graph of $(M, G)$. 
Hence, it is acceptable to leave out the adjective "abstract," when speaking of an "abstract GKM graph" or an "abstract GKM 1-skeleton."

Remark 2.9. In Definition 2.7, I weakened both Definition 2.1 and Proposition 2.4 by introducing $m_{e}$ and $\tau_{e, i}$, because, in general, the symplectic reduction of a manifold is an orbifold, and the reduction of an abstract 1-skeleton (see Section 4), built as the analogue of the symplectic reduction of a manifold, will be an abstract 1-skeleton and not a GKM 1-skeleton.

Definition 2.10. The abstract 1-skeleton $(\Gamma, \alpha)$ is $k$-independent if for all vertices $p$ and for all $k$-sets of neighbors $\left\{q_{1}, \ldots, q_{k}\right\}$ of $p$, the family $\left\{\alpha_{p, q_{1}}, \ldots, \alpha_{p, q_{k}}\right\}$ is linearly independent.

\section{CLASSICAL EXAMPLES OF GKM MANIFOLDS AND GRAPHS}

\subsection{The Grassmannian $G_{k, n}(\mathbb{C})$}

The Grassmannian $M=G_{k, n}(\mathbb{C})$ of complex $k$-planes in $\mathbb{C}^{n}$ is equipped with the action of the complex torus $T=\left(S^{1}\right)^{n}$ deduced from the natural action on $\mathbb{C}^{n}$.

This manifold has complex dimension $k(n-k)$, whereas the quotient of the torus by the kernel of the action is $(n-1)$-dimensional. Hence, if $k$ is different from 1 and $n-1$, conditions of Delzant's theorem are not fulfilled. Hence, the image of a moment map does not contain all data from the manifold.

Vertices of the GKM graph of $G_{k, n}(\mathbb{C})$ are in one-toone correspondence with $k$-subsets $S$ of $\{1, \ldots, n\}$. The vertex $p_{S}$ corresponds to the $k$-dimensional space generated by $e_{j}(j \in S)$. Two vertices $p_{S}$ and $p_{S^{\prime}}$ are adjacent if and only if $S \cap S^{\prime}$ has $k-1$ elements. Moreover the axial function is $\alpha_{p_{S}, p_{S^{\prime}}}=\sum_{i \in S^{\prime}} \theta_{i}-\sum_{j \in S} \theta_{j}$.

This graph has $\left(\begin{array}{l}n \\ k\end{array}\right)$ vertices, each of them linked with $k(n-k)$ adjacent vertices. Consequently, there are

$$
\frac{k(n-k)}{2}\left(\begin{array}{l}
n \\
k
\end{array}\right)=\frac{n(n-1)}{2}\left(\begin{array}{l}
n-2 \\
k-1
\end{array}\right)
$$

edges. We will often write $p_{S}=S$.

The projective space $\mathbb{P}^{n}(\mathbb{C})=G_{1, n+1}(\mathbb{C})$ is equipped with the effective action of the $n$-dimensional torus $\left(S^{1}\right)^{n+1} /\left(e^{i \theta}, \ldots, e^{i \theta}\right)$. The Fubini-Study form endows it with a Hamiltonian structure with moment map $\phi([z])(X)=-i\langle X(z), z\rangle /|z|^{2}\left(z \in \mathbb{C}^{n+1}, X \in \mathfrak{g}\right)$. This space satisfies the hypotheses of Delzant's theorem, thus the image of the moment map characterizes this space.
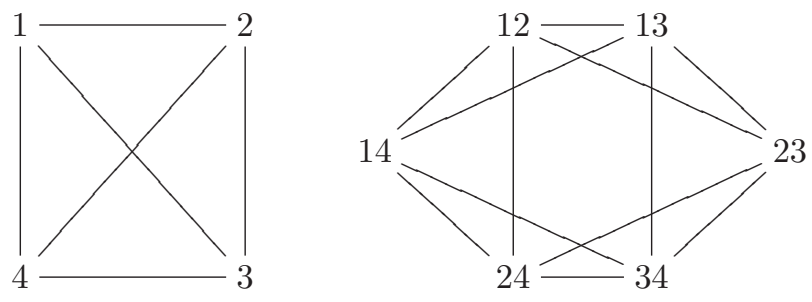

FIGURE 5. GKM graphs of $\mathbb{P}^{3}(\mathbb{C})$ and $G_{2,4}(\mathbb{C})$ (axial function omitted).

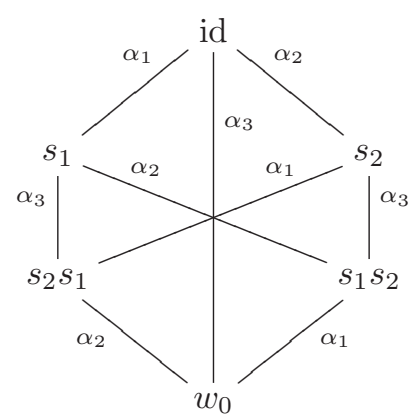

FIGURE 6. GKM graph of the flag manifold $G L(3, \mathbb{C}) / B$.

\subsection{The Flag Manifold $\mathrm{U}(\boldsymbol{n}) /\{$ diagonal matrices $\}$}

Let $K=U(n)$ be the group of $n \times n$ unitary matrices and $D$ be the subgroup of $K$ of diagonal unitary matrices. Denote by $M=U(n) / D \simeq G L(n, \mathbb{C}) / B$ the flag manifold of $G L(n, \mathbb{C})$, where $B$ is the Borel subgroup of $G L(n, \mathbb{C})$ of invertible upper triangular matrices. This manifold does not fulfill the hypotheses of Delzant's theorem, hence the GKM graph is valuable for characterizing this space.

The GKM graph of the flag manifold $G L(n, \mathbb{C}) / B$ is, in fact, the Cayley graph associated to the permutation group $S_{n}$. Vertices of $\Gamma$ are in one-to-one correspondence with permutations over $\{1, \ldots, n\}$. Two vertices $\sigma, \sigma^{\prime}$ are linked by an edge if and only if there exists a transposition $t_{i, j}=\left(\begin{array}{ll}i & j\end{array}\right)$ such that $\sigma^{\prime}=\sigma t_{i, j}$. Moreover $\alpha_{\sigma, \sigma t_{i, j}}$ equals $\theta_{j}-\theta_{i}$ if $\sigma(j)>\sigma(i)$, else $\theta_{i}-\theta_{j}$.

For example, let $\alpha_{1}=\theta_{2}-\theta_{1}, \alpha_{2}=\theta_{3}-\theta_{2}$, and $\alpha_{3}=\alpha_{1}+\alpha_{2}$. Then Figure 6 represents the GKM graph of $G L(3, \mathbb{C}) / B$.

\section{REDUCTION OF A 3-INDEPENDENT ABSTRACT 1-SKELETON}

Let $(\Gamma, \alpha)$ be a $d$-valent abstract 1 -skeleton. Here we assume that $(\Gamma, \alpha)$ is 3-independent (see Definition 2.10). 
When $(\Gamma, \alpha)$ is not 3 -independent we can still compute a reduction, but the result will be a hypergraph (see [Guillemin and Zara 00]). Although my programs can handle this case, I will not describe this similar theory here.

Fix a one-dimensional subtorus $H$ of $G$ with infinitesimal generator $\xi \in \mathfrak{g}$ lying in no $\operatorname{ker}\left(\alpha_{p, e}\right)$ (where $e=[p, q]$ runs over the set of edges). This vector $\xi$ gives rise to a graph orientation $o_{\xi}$ for $(\Gamma, \alpha)$, by saying that the edge $[p, q]$ satisfies $p<q$ if and only if $\alpha_{p, e}(\xi)<0$.

Assume that, for any codimension 2 subspace $\mathfrak{h}$ of $\mathfrak{g}$ and for any divalent subgraph $\Gamma_{\mathfrak{h}}$ that has edges $[p, q]$ such that $\alpha_{p, q} \in \mathfrak{h}^{\perp}$, each connected component $\Gamma_{0}$ of $\Gamma_{\mathfrak{h}}$ has exactly one maximum and one minimum (for the orientation $o_{\xi}$ ). We say that $\Gamma$ is strongly acyclic.

Let $f$ be a cohomology element of $(\Gamma, \alpha)$, that is $f$ : $\Gamma \longrightarrow \mathfrak{g}^{*}$ such that $f(q)-f(p)=\lambda_{e} \alpha_{p, e}$ for all edges $e=[p, q]$. We assume that $f$ is symplectic, that is, $\lambda_{e}>0$ for all $e$. The application $\phi=\phi_{f, \xi}: V_{\Gamma} \longrightarrow \mathbb{R}$ defined by $\phi(p)=\langle f(p), \xi\rangle$ satisfies $\frac{\phi(p)-\phi(q)}{\alpha_{q, e}(\xi)}>0$ for all $e=[p, q] ;$ we then say that $\phi$ is an $H$-moment for $(\Gamma, \alpha)$. Fix a regular value $c$ for $\phi$.

Let us build the reduced 1-skeleton, a graph $\Gamma_{c}$ with valence $d-1$ that will be an abstract 1 -skeleton with axial function vanishing on $\xi$. This corresponds to the fact that the reduction of a $2 d$-dimensional manifold $M$ is a $2(d-1)$-dimensional orbifold endowed with the action of $G / H$.

Vertices $S_{\Gamma_{c}}$ of $\Gamma_{c}$ are oriented edges $e=[p, q]$ such that $\phi(p)<c<\phi(q)$. For such an edge $e=[p, q]$, denote by $q_{i}$ all $(d-1)$ neighbors of $p$ other than $q$. Fix an index $i$. Let $\mathfrak{h}_{i}$ be the intersection of kernels of linear forms $\alpha_{p, q}$ and $\alpha_{p, q_{i}}$. The connected component $\Gamma_{0}$ of $\Gamma_{\mathfrak{h}_{i}}$ containing $p, q$, and $q_{i}$ is divalent, thanks to 3 -independence. By hypothesis $(\Gamma, \alpha)$ is strongly acyclic, hence there exist in $\Gamma_{0}$ exactly two edges cut by $c$, namely $e$ and another one, denoted by $e^{\prime}=\left[p^{\prime}, q^{\prime}\right]$. We then link vertices $[p, q]$ and

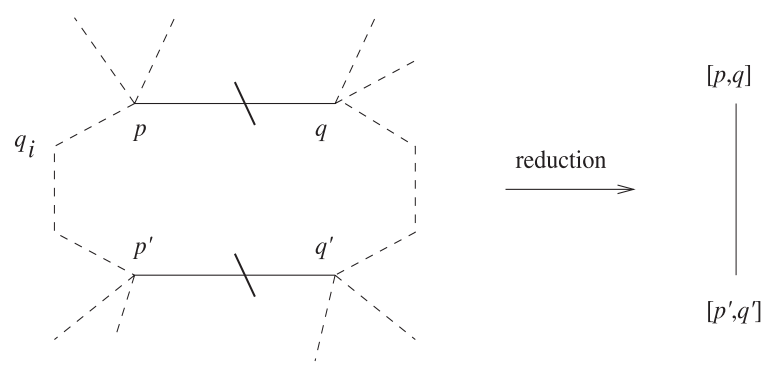

FIGURE 7. Reduction of an abstract 1-skeleton. $\left[p^{\prime}, q^{\prime}\right]$ by an edge in the reduction. See Figure 7. The axial function on the edge $\left[e, e^{\prime}\right]$ of $\Gamma_{c}$ is

$$
\alpha_{e, e^{\prime}}^{c}=\alpha_{p, q_{i}}-\frac{\alpha_{p, q_{i}}(\xi)}{\alpha_{p, q}(\xi)} \alpha_{p, q} .
$$

Notice that this is indeed a linear form vanishing on $\xi$.

Theorem 4.1. [Guillemin and Zara 01a] The graph $\left(\Gamma^{c}, \alpha^{c}\right)$ is an abstract 1 -skeleton with valence $(d-1)$. If $(\Gamma, \alpha)$ is $k$-independent, then $\left(\Gamma^{c}, \alpha^{c}\right)$ is $(k-1)$ independent.

When the abstract 1-skeleton $(\Gamma, \alpha)$ comes from a symplectic GKM manifold with Hamiltonian action, the reduction of $(\Gamma, \alpha)$ coincides with the GKM graph of the reduced manifold: $\Gamma(M)_{c}=\Gamma\left(M_{c}\right)$.

Remark 4.2. The reduction does not change when $c$ runs over a connected component of the set of regular points. Note also, that when we add a vector $\lambda_{0} \in \mathfrak{g}^{*}$ to $f$, all data are translated. Consequently, one can always consider the reduction at $c=0$.

Example 4.3. (Reductions of $\mathbb{P}^{4}(\mathbb{C})$.) $\quad$ Let $H$ be the onedimensional subtorus of $\left(S^{1}\right)^{4}$ with infinitesimal generator $\xi=(4,3,2,1,-10)$. Since the GKM graph of $\mathbb{P}^{4}(\mathbb{C})$ is 4-independent, its reductions are 3 -independent abstract GKM 1-skeleta. Choose $f(i)=\theta_{i}$ as a symplectic cohomology element. Critical values for $\phi=\phi_{f, \xi}$ are $4,3,2$, 1 , and -10 . In fact, we can restrict ourselves to reductions at $c=7 / 2,5 / 2,3 / 2$, and 0 . Reductions at $c=7 / 2$ and 0 are the same as at $c=5 / 2$ and $3 / 2$. See Figures 8 and 9 .

Example 4.4. (Reductions of $\boldsymbol{G L}(\mathbf{3}, \mathbb{C}) / \boldsymbol{B}$.) Since the GKM graph of $G L(3, \mathbb{C}) / B$ is not 3 -independent, its reductions are not graphs but hypergraphs. Let $H$ be generated by $\xi=(2,1,-3)$. Let $\alpha_{1}=\theta_{2}-\theta_{1}, \alpha_{2}=\theta_{3}-\theta_{2}$, and $\alpha_{3}=\alpha_{1}+\alpha_{2}$. Choose as a symplectic cohomology element the function $f$ defined by

$$
\begin{aligned}
f(\mathrm{id}) & =-\left(\alpha_{1}+\alpha_{2}\right), \\
f\left(s_{1}\right) & =-\alpha_{2}, \\
f\left(s_{2} s_{1}\right) & =\alpha_{1}, \\
f\left(w_{0}\right) & =\left(\alpha_{1}+\alpha_{2}\right), \\
f\left(s_{2}\right) & =-\alpha_{1}, \\
f\left(s_{1} s_{2}\right) & =\alpha_{2} .
\end{aligned}
$$

Critical values for $\phi=\phi_{f, \xi}$ are $\pm 5, \pm 4$, and \pm 1 . We can restrict ourselves to reductions at $c= \pm 3 / 2, \pm 9 / 2$, and 0 . Reductions at $c= \pm 3 / 2$ are the same as at $c= \pm 9 / 2$. 


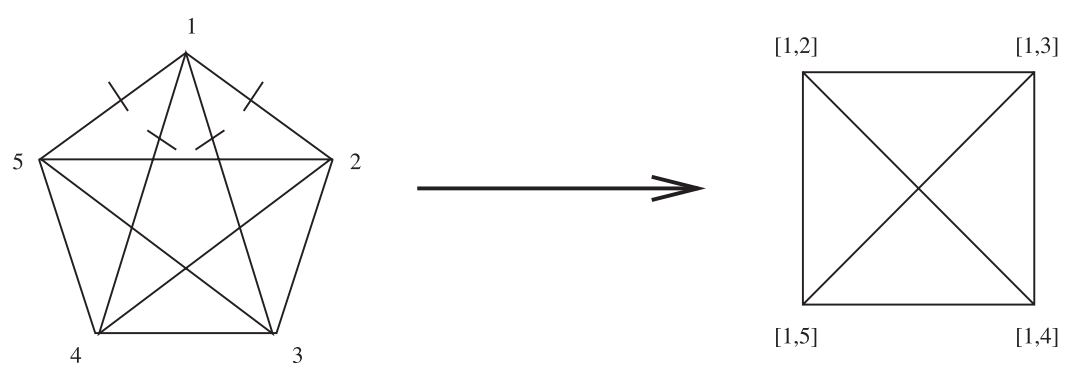

FIGURE 8. Reduction of $\mathbb{P}^{4}(\mathbb{C})$ along $\xi=(4,3,2,1,-10)$ and at $c=7 / 2$.
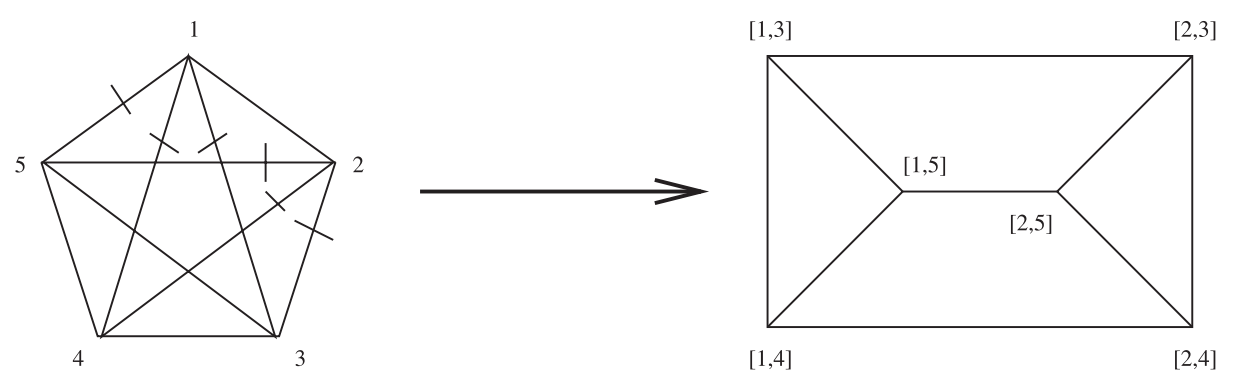

FIGURE 9. Reduction of $\mathbb{P}^{4}(\mathbb{C})$ along $\xi=(4,3,2,1,-10)$ and at $c=5 / 2$.
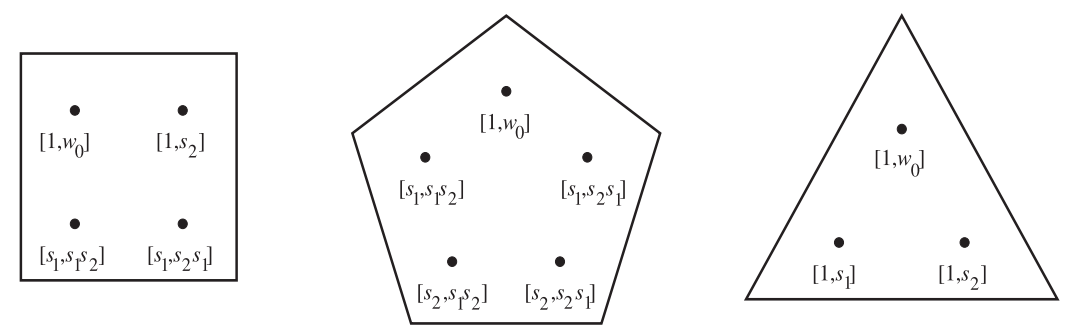

FIGURE 10. Reductions of $G L(3, \mathbb{C}) / B$ by $\xi=(2,1,-3)$ at $c=3 / 2, c=0$ and $c=9 / 2$.

In every case, the reduced graph has a unique hyperedge linking all vertices. See Figure 10.

\section{QUANTIZATION AND REDUCTION COMMUTE}

This section deals with the analogue of "quantization and reduction commute" for GKM graphs, as proved by Guillemin and Zara [Guillemin and Zara 01b] in the case of reduction by a one-dimensional torus.

Let $(\Gamma, \alpha)$ be a $d$-valent abstract 1-skeleton. Fix a onedimensional subtorus $H$ of $G$ with infinitesimal generator $\xi \in \mathfrak{g}$ lying in no $\operatorname{ker}\left(\alpha_{p, e}\right)$.

Let $f$ be a cohomology element for $(\Gamma, \alpha)$ with values in $\mathbb{Z}_{G}^{*}$. The application $\Theta(p)=e^{2 i \pi f(p)}$ is called a $K$ theory element for $(\Gamma, \alpha)$. The character of $\Theta$ is then defined by

$$
\chi(\Theta)=\sum_{p \in V_{\Gamma}} \frac{\Theta(p)}{\prod_{e \in E_{p}}\left(1-e^{2 i \pi \alpha_{p, e}}\right)} .
$$

In fact, the character $\chi(\Theta)$ has no pole [Guillemin and Zara 01b].

Assume that the abstract 1-skeleton is a GKM graph (that is, coming from a manifold) and that $f$ is symplectic $\left(\lambda_{e}>0\right.$ for all $\left.e\right)$. Let $\phi(p)=\langle f(p), \xi\rangle$ be the $H$-moment. Fix a regular value $c$ for $\phi$.

Guillemin and Zara proved that the invariant character $\chi(\Theta)^{H}$ (defined as the part of $\chi(\Theta)$ invariant under the action of $H$ ) can be expressed in terms of a GKM graph and $\Theta$, under the condensed form of a sum of rational fractions. This more precisely expressed in Theorem 5.1. 
Theorem 5.1. [Guillemin and Zara 01b] The reduced character $\chi_{c}(\Theta)$ is, by definition,

$$
\begin{aligned}
& \sum_{[p, q] \in \Gamma_{c}} \frac{1}{\left|\alpha_{p, q}(\xi)\right|} \times \\
& \sum_{\zeta^{\alpha_{p}, q(\xi)}=1} \frac{\zeta^{\langle f(p), \xi\rangle} e^{2 i \pi\left(f(p)-\frac{\langle f(p), \xi\rangle}{\alpha_{p, q}(\xi)} \alpha_{p, q}\right)}}{\prod_{r \in N(p) \backslash\{q\}}\left(1-\zeta^{\alpha_{p, r}(\xi)} e^{2 i \pi\left(\alpha_{p, r}-\frac{\alpha_{p, r}(\xi)}{\alpha_{p}, q(\xi)} \alpha_{p, q}\right)}\right)} .
\end{aligned}
$$

Then the invariant character equals the reduced character $\chi_{c=0}(\Theta)$.

Remark 5.2. In the case of the reduction of a Hamiltonian manifold, Meinrenken and Sjamaar [Meinrenken and Sjamaar 99] proved the equality $\chi_{c}(\Theta)=\chi(\Theta)^{H}$ at a regular value $c$ close to zero. Although $M_{c}$ and $\left(\Gamma^{c}, \alpha^{c}\right)$ change when $c$ crosses the critical value 0 , the character $\chi_{c}(\Theta)$ remains unchanged. This result is still true in the framework of examples that we will discuss in Section 6 . Hence in these examples, when 0 is not regular, we compute the reduced character at the regular value $c=1 / 10$ close enough to 0 .

Remark 5.3. Theorem 5.1 has two consequences. First, we have a condensed expression (sum of rational fractions) of $\chi(\Theta)^{H}$. In fact, when $(\Gamma, \alpha)$ comes from a Hamiltonian manifold $M$, the character of $Q(M)^{H}$ is huge. Second, the formula of Guillemin and Zara involves sums over roots of unity. When these roots are reasonable, computation is possible and does not explode when, for example, one dilates $\Theta$.

Example 5.4. (Case of $\mathbb{P}^{\mathbf{3}}(\mathbb{C})$.) Let $H$ be the one-dimensional torus generated by $\xi=(1,2,-1,-2)$. Choose $f_{\ell}(p)=\ell \theta_{p}(p=1, \ldots, 4)$ with $\ell \in \mathbb{N}^{*}$ and let $\Theta_{\ell}(p)=e^{2 i \pi f_{\ell}(p)}$. Let $u_{j}=e^{2 i \pi \theta_{j}}(j=1, \ldots, 4)$. Hence the invariant character is $\chi\left(\Theta_{\ell}\right)^{H}=\sum u_{1}^{\ell_{1}} u_{2}^{\ell_{2}} u_{3}^{\ell_{3}} u_{4}^{\ell_{4}}$, where the sum is over the set of integers $\left(\ell_{1}, \ell_{2}, \ell_{3}, \ell_{4}\right) \in$ $\mathbb{N}^{4}$ such that $\ell_{1}+\ell_{2}+\ell_{3}+\ell_{4}=\ell$ and $\ell_{1}+2 \ell_{2}=\ell_{3}+2 \ell_{4}$. The result is a polynomial in $u_{j}$ with the number of monomials quadratic in $\ell$. For example $\chi\left(\Theta_{500}\right)^{H}$ has 21001 monomials spreading over 93 pages.

On the other hand, one can check that Theorem 5.1 leads to a condensed formula in which size does not vary when $\ell$ grows. For example, $\chi_{0}\left(\Theta_{6 m}\right)$ equals

$$
\begin{aligned}
& \frac{u_{2}^{2 m+1} u_{3}^{4 m+2}\left(u_{2} u_{3}^{3}+u_{1}^{2} u_{3} u_{4}+u_{1} u_{2} u_{4}^{2}\right)}{\left(u_{2}^{2} u_{3}-u_{1}^{3}\right)\left(u_{3}^{4}-u_{2} u_{4}^{3}\right)} \\
+ & \frac{u_{2}^{3 m+1} u_{4}^{3 m+1}\left(u_{2}^{3} u_{4}^{3}+u_{1} u_{2} u_{3} u_{4}+u_{1}^{2}+u_{1}^{2} u_{2} u_{3}^{2} u_{4}+u_{1}^{3} u_{3}^{3}\right)}{\left(u_{2}^{3} u_{4}-u_{1}^{4}\right)\left(u_{2} u_{4}^{3}-u_{3}^{4}\right)}
\end{aligned}
$$

$$
\begin{aligned}
& +\frac{u_{1}^{4 m+2} u_{4}^{2 m+1}\left(u_{1}^{3} u_{4}+u_{2}^{2} u_{3} u_{4}+u_{1} u_{2} u_{3}^{2}\right)}{\left(u_{1}^{4}-u_{2}^{3} u_{4}\right)\left(u_{1} u_{4}^{2}-u_{3}^{3}\right)} \\
& +\frac{u_{1}^{3 m+2} u_{3}^{3 m+2}\left(u_{1} u_{3}+u_{2} u_{4}\right)}{\left(u_{1}^{3}-u_{2}^{2} u_{3}\right)\left(u_{3}^{3}-u_{1} u_{4}^{2}\right)} .
\end{aligned}
$$

Formulas for $\chi_{0}\left(\Theta_{6 m+k}\right)(1 \leq k \leq 5)$ are similar.

Remark 5.5. A direct computation of the invariant character leads to the enumeration of indices satisfying linear conditions of the form $\sum_{j} a_{j} \ell_{j}=T(\ell)$ (for an affine operator $T$ ) intractable problem when $\ell$ grows.

Remark 5.6. In the case of toric manifolds, the reduced manifold is still toric. Brion's formulas [Brion and Vergne 97, Barvinok 02] have been recently implemented in polynomial time with Barvinok's algorithm by the LattE team [De Loera et al. 04].

\section{PROGRAMS}

This section describes my programs reduction.mws and character.mws, computing respectively the reduction of an abstract 1-skeleton and the reduced character of a $K$-theory element of a GKM 1-skeleton. Recall that graph reduction needs an abstract 1-skeleton $(\Gamma, \alpha)$ (plus other hypotheses). It returns a graph (more precisely an abstract 1-skeleton) if $(\Gamma, \alpha)$ is 3 -independent, otherwise it returns a hypergraph. On the other hand, character reduction needs a GKM 1-skeleton but is independent of the notion of 3 -independence.

\subsection{Data Storage and Program Handling}

Fix a numbering $p_{1}, \ldots, p_{N}$ of vertices of the abstract 1-skeleton. The set of vertices is represented by the list $S=\left(p_{1}, \ldots, p_{N}\right)$. Edges and axial function are stored in the $N \times N$ matrix $A$ such that $a_{i, j}=\alpha_{p_{i}, p_{j}}$ if $p_{i}$ and $p_{j}$ are adjacent, else $a_{i, j}=0$. The matrix $A$ is the generalized adjacence matrix of $(\Gamma, \alpha)$ (for this numbering). This matrix allows an efficient check for adjacence, cycles, connected components, etc.

Hence, an abstract 1-skeleton is represented by the matrix $A$ and the list $S$.

The cohomology element $f$ is encoded by the list $F=\left(f\left(p_{1}\right), \ldots, f\left(p_{N}\right)\right)$. Vectors and linear forms are represented in the canonical basis of $\mathbb{R}^{n}$; hence, the evaluation of a form on a vector corresponds to a scalar product.

Example 6.1. Let us fix the numbering $p_{1}=1, p_{2}=2$, $p_{3}=3$ of vertices of the GKM graph of $\mathbb{P}^{2}(\mathbb{C})$; hence, 
$S=(1,2,3)$. Since $\alpha_{p_{i}, p_{j}}=\theta_{j}-\theta_{i}$, the generalized adjacence matrix is

$$
\left(\begin{array}{ccc}
0 & (-1,1,0) & (-1,0,1) \\
(1,-1,0) & 0 & (0,-1,1) \\
(1,0,-1) & (0,1,-1) & 0
\end{array}\right)
$$

The cohomology element $f(p)=\theta_{i}$ is represented by $F=$ $((1,0,0),(0,1,0),(0,0,1))$. Suitable $\xi$ and regular value $c$ are, for example, $(2,1,-3)$ and $3 / 2$.

My two programs are easy to use: the user only provides the 1-skeleton and reduction or character data, under the form $S, A, F, \xi, c$. This can be done either manually, or with procedures generating classical examples (Grassmannian, $G L(3, \mathbb{C}) / B$, cycle with $4 N$ vertices, product of graphs). For example, consider the reduction of $G_{2,4}(\mathbb{C})$ by the torus of infinitesimal generator $\xi=(3,2,1,-6)$, at the regular value $c=0$. Then the command lines building data associated to this reduction as well as the cohomology element $f(p)=5 \theta_{p}$ inducing the moment map are described below.

$$
\begin{aligned}
& \mathrm{S}:=\operatorname{Sgrass}(2,4) \\
& \mathrm{A}:=\operatorname{Agrass}(2,4) \\
& \mathrm{F}:=\operatorname{Fgrass}(2,4,5) \\
& \mathrm{xi}:=[3,2,1,-6] \\
& \mathrm{C}:=0
\end{aligned}
$$

Command lines for computing vertices, cohomology element, and generalized adjacence matrix of the reduced graph are described below. The last line gives a graphical representation of the graph.

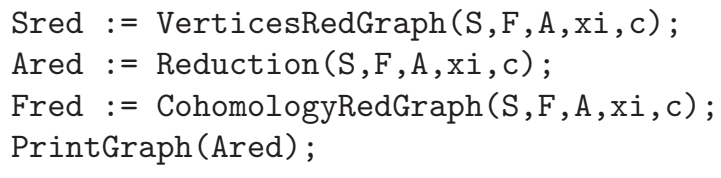

On the other hand, the command line for the reduced character is

$$
\mathrm{P}:=\text { CharacterRed }(\mathrm{S}, \mathrm{F}, \mathrm{A}, \mathrm{xi}, \mathrm{C}) \text {; }
$$

Both programs (for reduction and character computation) contain internal checks pointing out to the user the possible failure of any condition on input data (see Proposition 2.4, Definition 2.7, Sections 4 and 5).

\subsection{Reduction of a 3-Independent Abstract GKM 1-Skeleton}

The first step of the program reduction.mws is to find all vertices of the reduced graph, that is, all edges $e=[p, q]$ with $\phi(p)<c<\phi(q)$. Let us fix such an edge $e=[p, q]$ and let $r$ be a neighbor of $p$ different from $q$.

Three-independence implies that there exists a unique neighbor $s$ of $r$ other than $p$ such that the triple of vectors $\left\{\alpha_{q, p}, \alpha_{p, r}, \alpha_{r, s}\right\}$ has rank 2 (see Figure 7$)$. Finding this $s$ is performed by the procedure NeighborDimTwo $(A, q, p, r)$; its implementation is straightforward.

The algorithm computing the reduction of a 3-independent abstract 1-skeleton is:

For all vertex $e=[p, q]$ of the reduced 1-skeleton

for all neighbor $r$ of $p$ other than $q$ compute the reduced axial function find the only neighbor $s$ of $r$ other than $p$... ...such that $\left\{\alpha_{q, p}, \alpha_{p, r}, \alpha_{r, s}\right\}$ has rank 2 repeat

if $[r, s]$ is cut by $c$

then link $[p, q]$ and $[r, s]$ in the reduced... ... 1-skeleton quit the "repeat" loop else $q \longleftarrow p$

$$
\begin{aligned}
& p \longleftarrow r \\
& r \longleftarrow s
\end{aligned}
$$

end if

end of "repeat"

end of loop over neighbors of $p$ end of loop over vertices of the reduced graph

Note that strong acyclicity ensures us that the program will never be trapped in an infinite "repeat" loop.

\subsection{Computing the Invariant Character as a Sum of Rational Fractions}

The difficult part of character.mws is not implementing formulas, but finding an efficient way to do it. A raw procedure leads to computations that explode, even for small examples. More precisely, we need to compute sums appearing in Theorem 5.1;

$$
S\left(m ; k, k_{1}, \ldots, k_{d}\right)=\sum_{\ell=1}^{m} \frac{\zeta^{\ell k}}{\left(1-\zeta^{\ell k_{1}} U_{1}\right) \cdots\left(1-\zeta^{\ell k_{d}} U_{d}\right)},
$$

where $\zeta$ is the $m$ th root of unity $e^{\frac{2 i \pi}{m}}$ and $U_{j}$ are indeterminates. Using a formal series development, one easily obtains an expression that may be used (see [Zagier 73]);

$S\left(m ; k, k_{1}, \ldots, k_{d}\right)=\frac{\sum_{s_{1}, \ldots, s_{d}=0}^{m-1} a_{s_{1}, \ldots, s_{d}} U_{1}^{s_{1}} \cdots U_{d}^{s_{d}}}{\left(1-U_{1}^{m}\right) \cdots\left(1-U_{d}^{m}\right)}$, 


\begin{tabular}{|c||c|c|c|}
\hline Graph of the manifold & Dim. effective torus & Valence & Number of vertices \\
\hline \hline $\mathbb{P}^{2}(\mathbb{C})$ & 2 & 2 & 3 \\
\hline$G L(3, \mathbb{C}) / B$ & 2 & 3 & 6 \\
\hline$G_{2,4}(\mathbb{C})$ & 3 & 4 & 6 \\
\hline $\mathbb{P}^{5}(\mathbb{C})$ & 5 & 5 & 6 \\
\hline$G_{2,5}(\mathbb{C})$ & 4 & 6 & 10 \\
\hline $\mathbb{P}^{4}(\mathbb{C}) \times G L(3, \mathbb{C}) / B$ & 6 & 7 & 30 \\
\hline $\mathbb{P}^{8}(\mathbb{C})$ & 8 & 8 & 9 \\
\hline$G_{3,6}(\mathbb{C})$ & 5 & 9 & 20 \\
\hline$G_{2,4}(\mathbb{C}) \times G_{2,5}(\mathbb{C})$ & 7 & 10 & 60 \\
\hline $\mathbb{P}^{20}(\mathbb{C})$ & 20 & 20 & 21 \\
\hline$G_{5,10}(\mathbb{C})$ & 9 & 25 & 252 \\
\hline$G_{6,13}(\mathbb{C})$ & 12 & 42 & 1716 \\
\hline
\end{tabular}

TABLE 1. GKM graphs of classical manifolds.

where $a_{s_{1}, \ldots, s_{d}}$ equals $n$ when $m$ divides $k+k_{1} s_{1}+\cdots+$ $k_{d} s_{d}$, else 0 .

Marc A. A. van Leeuwen has performed an efficient recursive implementation of this formula, in the procedure SumRootUnit ( $\mathrm{k}, \mathrm{L}, \mathrm{X}, \mathrm{U}, \mathrm{m})$. Here $L$ is the list of constants $k_{j}$ and $X$ is an intermediary variable. The algorithm of the procedure is:

let $d$ be the cardinal of $\mathrm{L}$

let recur $($ level,s) be a local procedure defined by

if level is strictly greater than $d$

then return $X^{s} \bmod m$

else return the sum for $t=0, \ldots, m-1$ of. .

$\ldots U_{\text {level }}^{t} * \operatorname{recur}\left(\right.$ level+1, $\left.\mathrm{s}-\mathrm{t} * L_{\text {niv }}\right)$

end of local procedure

let $R$ be the coefficient of the polynomial. .

$\ldots r$ recur $(1,0)$ in $X$ of degree $k \bmod m$

return $m \times R$ divided by the product of $\left(1-U_{k_{j}}\right), \ldots$

... with $j$ ranging from 1 to $d$.

This procedure returns the sum of $\left|\Gamma_{0}\right|$ rational fractions. The dilatation of the cohomology element by a factor $\ell$ - even of the order of billion-does not cost more time or memory. Only a monomial factor before each fraction grows, while the result under polynomial form explodes (see Example 5.4).

\section{TEST OF PROGRAMS}

Let me begin with several general remarks.

I stress that I rewrote Maple procedures on vectors: addition, multiplication by a constant, scalar product, test of colinearity, rank of three vectors, etc. This greatly increased the speed of the programs. Moreover, using lists instead of vectors has gained a lot of time. Finally, encoding the null vector (corresponding to two nonadjacent vertices) by the integer 0 in the generalized adjacence matrix allowed a fast test of edges. More generally, great care towards efficiency was taken during the implementation.

Here, I describe tests on classical examples of GKM graphs coming from Grassmannians, from the flag manifold $G L(3, \mathbb{C}) / B$, and their products. In Table 1 , one can find for several classical manifolds: dimension of effective torus acting, valence, and number of vertices of the graph.

For the Grassmannian $G_{n, k}(\mathbb{C})$, the cohomology element is $f\left(p_{S}\right)=\sum_{s \in S} \theta_{s}$ (see Example 4.3). For the flag manifold $G L(3, \mathbb{C}) / B$ the cohomology element is described in Example 4.4. Denote by $\xi_{r}$ the vector $(r-1, \ldots, 1,-r(r-1) / 2) \in \mathbb{C}^{r}$. In my examples I always reduced at the regular value $c=1 / 10$ ( Recall that the valence $d$ of the GKM 1-skeleton equals the dimension of the underlying manifold. Moreover, the reduced graph has valence $d-1$.

Tests were performed on standard 1.13-GHz computer, running Maple 9. I checked retro-compatibility down to Maple Vr5.

Note that for graph reduction most of the computation time is spent checking colinearity of two vectors and checking the rank of a system of three vectors. Improving, even slightly, the speed of these two tasks would lead to a substantial time savings. Nevertheless, the reduction of 25-valent graphs with hundreds of vertices is already done in a very competitive time. See Table 2.

Now come remarks on character reduction. The procedure SumRootUnit $(\mathrm{k}, \mathrm{L}, \mathrm{X}, \mathrm{U}, \mathrm{m})$, computing recursively sums of roots of unity and described in Section 6.3, is really efficient. Its two main parameters (directly influencing computation time) are the valence of the graph 


\begin{tabular}{|c||c|c|c|c|}
\hline $\begin{array}{c}\text { Manifold } \\
\text { to be reduced }\end{array}$ & $\begin{array}{c}\text { Generator } \xi \\
\text { of } H\end{array}$ & $\begin{array}{c}\text { Nb. of vertices } \\
\text { of reduced graph }\end{array}$ & $\begin{array}{c}\text { Nb. of (hyper)edges } \\
\text { of reduced graph }\end{array}$ & $\begin{array}{c}\text { Computation } \\
\text { time }\end{array}$ \\
\hline \hline $\mathbb{P}^{2}(\mathbb{C})$ & $\xi_{3}$ & 2 & 1 & $<0.1 \mathrm{sec}$ \\
\hline$G L(3, \mathbb{C}) / B$ & $(1,0,-1)$ & 5 & 1 & $<0.1 \mathrm{sec}$ \\
\hline$G_{2,4}(\mathbb{C})$ & $\xi_{4}$ & 6 & 10 & $<0.1 \mathrm{sec}$ \\
\hline $\mathbb{P}^{5}(\mathbb{C})$ & $\xi_{6}$ & 5 & 18 & $<0.1 \mathrm{sec}$ \\
\hline $\mathbb{P}^{5}(\mathbb{C})$ & $(3,2, \ldots,-3)$ & 9 & 30 & $0.1 \mathrm{sec}$ \\
\hline$G_{2,5}(\mathbb{C})$ & $(3,2,1,0,-6)$ & 12 & 45 & $11.6 \mathrm{sec}$ \\
\hline $\mathbb{P}^{4} \times G L(3) / B$ & $\xi_{8}$ & 20 & 70 & $0.2 \mathrm{sec}$ \\
\hline $\mathbb{P}^{8}(\mathbb{C})$ & $(4,3, \ldots,-4)$ & 20 & 148 & $0.4 \mathrm{sec}$ \\
\hline$G_{3,6}(\mathbb{C})$ & $(3,2, \ldots,-3)$ & 37 & 324 & $9.8 \mathrm{sec}$ \\
\hline$G_{2,4} \times G G_{2,5}$ & $\xi_{9}$ & 72 & 190 & $2.2 \mathrm{sec}$ \\
\hline $\mathbb{P}^{20}(\mathbb{C})$ & $\xi_{21}$ & 20 & 7560 & $84.7 \mathrm{sec}$ \\
\hline$G_{5,10}(\mathbb{C})$ & $\xi_{10}$ & 630 & 113652 & $8113.0 \mathrm{sec}$ \\
\hline$G_{6,13}(\mathbb{C})$ & $\xi_{13}$ & 5544 & & \\
\hline
\end{tabular}

TABLE 2. Reduction of GKM graphs of classical manifolds.

\begin{tabular}{|c||c|c|c|c|}
\hline $\begin{array}{c}\text { Manifold } \\
\text { to be reduced }\end{array}$ & $\begin{array}{c}\text { Generator } \xi \\
\text { of } H\end{array}$ & $\begin{array}{c}\text { Nb. of vertices } \\
\text { of reduced graph }\end{array}$ & $\begin{array}{c}\text { Order of } \\
\text { roots of unity }\end{array}$ & $\begin{array}{c}\text { Computation } \\
\text { time }\end{array}$ \\
\hline \hline $\mathbb{P}^{2}(\mathbb{C})$ & $\xi_{3}$ & 2 & 4 and 5 & $<0.1 \mathrm{sec}$ \\
\hline$G L(3, \mathbb{C}) / B$ & $(1,0,-1)$ & 5 & 1 and 2 & $<0.1 \mathrm{sec}$ \\
\hline$G_{2,4}(\mathbb{C})$ & $\xi_{4}$ & 6 & 7 to 9 & $0.1 \mathrm{sec}$ \\
\hline $\mathbb{P}^{5}(\mathbb{C})$ & $\xi_{6}$ & 5 & 16 to 20 & $22.0 \mathrm{sec}$ \\
\hline $\mathbb{P}^{5}(\mathbb{C})$ & $(3,2, \ldots,-3)$ & 9 & 2 to 6 & $0.2 \mathrm{sec}$ \\
\hline$G_{2,5}(\mathbb{C})$ & $(3,2,1,0,-6)$ & 12 & 6 to 9 & $16.9 \mathrm{sec}$ \\
\hline $\mathbb{P}^{8}(\mathbb{C})$ & $(4,3, \ldots,-4)$ & 20 & 1 to 8 & $743.0 \mathrm{sec}$ \\
\hline$G_{3,6}(\mathbb{C})$ & $(3,2, \ldots,-3)$ & 37 & 1 to 6 & $891.4 \mathrm{sec}$ \\
\hline
\end{tabular}

TABLE 3. Computation of the reduced character of a $K$-theory element of classical manifolds.

and the values $\alpha_{p, q}(\xi)$ involved in Theorem 5.1. On one hand, the valence changes the depth of recursivity. On the other hand, sums are over roots of unity of order $\alpha_{p, q}(\xi)$, where $[p, q]$ runs over all vertices of the reduced graph.

However, for every reduced character computation, we need to call this procedure for each vertex of the reduced graph. Each call to the procedure gives a rational fraction appearing in the result. Hence, computing a reduced character is quite expensive when the reduced graph has many vertices.

For a fixed manifold, it is interesting to see what happens when we change the torus $H$. This influences the number of sums of roots of unity that need to be evaluated and the nature of the roots of unity. See Table 3 for examples of computations of the reduced character of a $K$-theory element of a GKM graph.

\section{ACKNOWLEDGMENTS}

The author thanks the referee for his/her careful work and judicious remarks.

\section{REFERENCES}

[Atiyah and Bott 84] M. F. Atiyah and R. Bott. "The Moment Map and Equivariant Cohomology." Topology 23:1 (1984), 1-28.

[Audin 91] Michèle Audin. The Topology of Torus Actions on Symplectic Manifolds. Basel: Birkhäuser Verlag, 1991.

[Barvinok 02] Alexander Barvinok. A Course in Convexity, Graduate Studies in Mathematics, 54. Providence, RI: American Mathematical Society, 2002.

[Berline and Vergne 82] Nicole Berline and Michèle Vergne. "Classes caractéristiques équivariantes. Formule de localisation en cohomologie équivariante." C. R. Acad. Sci. Paris Sér. I Math. 295:9 (1982), 539-541.

[Brion and Vergne 97] Michel Brion and Michèle Vergne. "Residue Formulae, Vector Partition Functions and Lattice Points in Rational Polytopes." J. Amer. Math. Soc. 10:4 (1997), 797-833.

[De Loera et al. 04] Jesús A. De Loera, Raymond Hemmecke, Jeremiah Tauzer, and Ruriko Yoshida. "Effective Lattice Point Counting in Rational Convex Polytopes." J. Symbolic Comput. 38:4 (2004), 1273-1302. 
[Delzant 88] Thomas Delzant. "Hamiltoniens périodiques et images convexes de l'application moment." Bull. Soc. Math. France 116:3 (1988), 315-339.

[Demazure 70] Michel Demazure. "Sous-groupes algébriques de rang maximum du groupe de Cremona." Ann. Sci. École Norm. Sup.(4) 3 (1970), 507-588.

[Goresky et al. 98] Mark Goresky, Robert Kottwitz, and Robert MacPherson. "Equivariant Cohomology, Koszul Duality, and the Localization Theorem." Invent. Math. 131:1 (1998), 25-83.

[Guillemin 94] Victor Guillemin. Moment Maps and Combinatorial Invariants of Hamiltonian $T^{n}$-spaces, Progress in Mathematics, 122. Boston, MA: Birkhäuser Boston Inc., 1994.

[Guillemin and Zara 99] Victor Guillemin and Catalin Zara. "Equivariant de Rham Theory and Graphs." Asian J. Math. 3:1 (1999), 49-76.
[Guillemin and Zara 00] Victor Guillemin and Catalin Zara. "Morse Theory on Graphs." math.CO/0007161, 2000.

[Guillemin and Zara 01a] Victor Guillemin and Catalin Zara. "1-Skeleta, Betti Numbers, and Equivariant Cohomology." Duke Math. J. 107:2 (2001), 283-349.

[Guillemin and Zara 01b] Victor Guillemin and Catalin Zara. "G-Actions on Graphs." Internat. Math. Res. Notices 2001:10 (2001), 519-542.

[Jeffrey and Kirwan 95] Lisa C. Jeffrey and Frances C. Kirwan. "Localization for Nonabelian Group Actions." Topology 34:2 (1995), 291-327.

[Meinrenken and Sjamaar 99] Eckhard Meinrenken and Reyer Sjamaar. "Singular Reduction and Quantization." Topology 38:4 (1999), 699-762.

[Zagier 73] Don Zagier. "Higher Dimensional Dedekind Sums." Math. Ann. 202 (1973), 149-172.

Charles Cochet, Institut de Mathématiques de Jussieu, Université Paris 7, UFR de mathématiques, UMR 7586, case 7012, 2 place Jussieu, 75251 Paris cedex 05, France (cochet@math.jussieu.fr) http://www.math.jussieu.fr/ cochet/

Received April 29, 2004; accepted September 23, 2004. 